\title{
Spatial data infrastructures for cities in developing countries
}

\section{Lessons from the Bangkok experience}

\author{
Ian D. Bishop, Francisco J. Escobar, Sadasivam Karuppannan, \\ Ian P. Williamson* and Paul M. Yates \\ Department of Geomatics, The University of Melbourne, VIC 3010, Australia
}

\section{Ksemsan Suwarnarat}

Policy and Planning Unit, Bangkok Metropolitan Administration, Bangkok, Thailand

\author{
Haider W. Yaqub \\ Planner, City of Islamabad, Pakistan
}

\begin{abstract}
The cities of the developing world face major problems in managing growth and their urban infrastructure. The experiences of cities in the developed world have usually proved inappropriate in developing countries and consequently new and innovative solutions are continually being explored for these cities. Geographic information systems and the underlying spatial data infrastructures appear to offer significant potential to assist in managing human settlements in developing countries. In examining the status of spatial data infrastructures in developing countries, the first part of the paper compares cities in developing countries to those in the developed world. It then highlights issues facing developing countries in establishing spatial data infrastructures to support efficient urban land management. The paper describes the Bangkok Land Information System as an example of a city attempting to use spatial information technologies to manage the urban environment. This case study, together with the experience of the authors, are used to describe generic issues involved in the development of spatial data infrastructures for cities in similar socio-economic conditions. The paper provides a technical and institutional framework for the development of spatial data infrastructures for cities in developing countries. The paper concludes by suggesting that simple, low cost, project oriented, easily maintained and user-friendly spatial information technologies have the best chance of success. $\odot 2000$ Elsevier Science Ltd. All rights reserved
\end{abstract}

Keywords: Spatial data infrastructures, Geographic information systems, Developing countries, Urban, Sustainable development

\section{Introduction}

The urban population worldwide is growing at a much faster rate than the population as a whole, and by larger increments than ever before. Improving social and economic conditions for all people and promoting

*Corresponding author. Tel: + 61-3-8344-4431; fax: + 61-3-93474128; e-mail: i.williamson@eng.unimelb.edu.au sustainable development is increasingly an urban challenge. As cities grow, these improvements become increasingly complex (United Nations, 1995). The world's urban population in 1996 was estimated at 2.6 billion. Within 10 years more than half of the world population will be in cities, 3.3 billion of the 6.59 billion total. Urban areas are globally expected to double to more than 4 billion people by 2025 , some $80 \%$ of them in developing countries. 
These population changes have a profound effect on the demand for services, not only in large urban agglomerations - mega cities - but just as importantly in intermediate and smaller cities. To sustain these cities there will be a high demand for resources and services from within the urban and surrounding areas and an urgent demand to improve the management and delivery of the services. To meet this demand, systems and procedures will need to be developed that assist in providing the required resources and management in a more efficient and effective manner. Unfortunately, many of the current methods and approaches to managing cities in the developed world have proven only partly successful in developing countries. Different and innovative approaches are required to solve the urgent problems facing Third World cities. However, some of the good old methods may still be relevant because to some extent, today's conditions of cities in developing countries are similar to cities in developed countries some 50 or 80 years ago but not exactly the same. This paper will evaluate the wide range of spatial information technologies and tools available today which promise considerable potential in supporting these requirements.

Over the last decade cities in developing countries have enthusiastically embraced geographic information systems (GIS) (the business systems or applications) and the supporting spatial data infrastructures (SDI) (the geodetic control and common base maps). As a result, the experience available to developing countries is based almost solely on the experiences of cities in developed countries. This experience shows that where geographical aspects are important for management, decision making and planning, these GISs and SDIs have helped to improve the efficiency of these urban management activities. Typically, more than $90 \%$ of information required for a city's administration has a spatial component, such as parcels of land, road networks, utility infrastructure, emergency services, garbage collection and recreational areas with the result that GIS have been seen as an essential technology for urban management.

In contrast, for most cities of the developing world such spatial information related activities are undertaken manually if at all. These cities are mostly larger in population and area than in the developed world, are rapidly expanding and hence are very anxious to adopt any technologies which promise to make the management of the cities more effective and efficient. However, the institutional environment in these cities to allow the adoption of GIS is very different from the cities in the developed world. The objective of this paper therefore is to explore the potential of applying spatial information technologies in the management of cities in developing countries.

First the distinguishing characteristics of cities in developing countries as compared to those in developed countries is explored with a particular focus on spatial information requirements. Second the status of spatial data infrastructures in developing countries is discussed (recognizing that without some SDI it is almost impossible to introduce GIS in any city) and third a detailed case study is made of the use of spatial information technologies in the City of Bangkok. The paper concludes by drawing on the Bangkok experience, as well as the experience of the authors in other cities in developing countries, to summarize and highlight issues and possible strategies concerned with the use of GIS in cities in developing countries.

\section{Cities in developing countries}

\section{Distinguishing characteristics}

Numerous differences exist between the cities of developing countries and those in the developed world. In particular, differences exist in their ability to adopt GIS technologies. Some important distinguishing characteristics of the cities in developing countries which affect their ability to adopt spatial information technologies include:

(1) The rapid growth in population is not matched by growth in delivery of land for housing, services, utilities and infrastructure important to sustain a reasonable quality of life. This is evident from the sprawl of informal settlements, increase in congestion, air and water pollution, poor and deteriorating infrastructure, and dilapidated housing (Williamson, 1991).

(2) The growth of cities is dictated by market forces rather than strategic planning. Urban development is often uncoordinated and land speculation pursues quick financial gains. The suburban and rural areas are invaded by market-induced developments.

(3) Laws and guidelines for land registration, planning and land management is diverse and often uncoordinated in developing countries. The reasons are many and varied between different countries with different traditions and political structures. Thus, the establishment of SDI cannot be easily standardized for developing countries.

(4) Developed countries in general have moved from a prescriptive form of land use planning (e.g. Master Plan) towards a market oriented "spotzoning" approach conforming to environmental guidelines. However prescriptive urban land use planning is adopted in most of developing countries, resulting in long term land use and master plans which are less market sensitive and consequently often not followed.

(5) In order to cater for an urgent demand for housing, almost all cities in developing countries have a significant proportion of the population living as squatters in slums or informal settlements. As a result tenure and ownership is often obscured and unregulated in the cities of developing coun- 
tries with access to land and security of tenure being major problems. Planning and the management of services is very poor or non existent in these circumstances. Since these informal settlements are a special phenomenon in cities of developing countries, the strategies for managing cities in the developed world are inappropriate to these circumstances.

(6) Unplanned developments make it difficult to provide utilities, while providing utilities at a later stage is very costly. As a result of poor systems for land administration and either poor or non existent base mapping, city administrations usually do not know the location of existing services. It follows that efficient maintenance of services and infrastructure is almost impossible. Without proper land information, the acquisition of land for public facilities or the undertaking of any city planning exercise is very cumbersome, if not impossible.

(7) There is little or no spatial information infrastructure (and particularly large scale base maps). The biggest single barrier stopping the construction of a spatial infrastructure is a lack of data. Other limitations include inefficient processes for purchasing spatial information or GIS technology; lack of skilled personnel to establish and manage the infrastructure; and lack of funding or political will to support the construction of the infrastructure.

In addition to these specific characteristics, there is a growing expectation within the developed world, that cities in the developing world will meet externally prescribed standards in terms of environmental protection and working conditions. These external constraints imply improved information flow to administer effective plans for urban management, resource allocation and distribution (Lai and Wong, 1996). The lessons from the developed world are that to do any progressive planning of these cities it is necessary to develop a spatial information system which can be an efficient tool for management of their urban resources. The development of such spatial data infrastructures and the resulting GIS which can build upon them are considered an essential requirement for the cities of the developed world to improve their management. The status of SDIs in developing countries is summarized below.

\section{Spatial data infrastructures in developing countries}

The availability of spatial information for cities in developing countries is poor or non existent. In many cases, the spatial data is in the form of unscaled sketches. Where maps exist they are often out dated or classified as restricted information and access by public departments is very difficult if not impossible. Where current and unclassified maps exist, they are usually of different scales aggravating the problem of sharing information efficiently among various sectors of the city. Digital representation of spatial data is even rarer due to the lack of appropriate equipment and trained staff. Few cities are ready to begin generating digital spatial databases as the task is seen to be too large, too costly and too complex both technically and administratively. The design and implementation of a workable spatial data infrastructure is often a dream for the future, and without a SDI, GIS are not possible.

Reproduction of maps, where they exist, is often done with ammonia printers with the original maps prepared and up-dated on transparent sheets. Cadastral data is usually stored in hard copy registers and updated manually where available. Where a digital database is being prepared for a city, map production often continues to be done manually in parallel. Information about state owned land or government property is poor. In many cities, there are large military land holdings where information is restricted.

Often maps showing land ownership are in the form of sketches which are not to scale. Therefore, the exact demarcation of much of the land is impossible or very difficult and time consuming. Most information about land ownership and utilities are in the experienced hands of key personnel in separate departments. Due to the absence of any other information system they are treated as the primary information source. As a result this information can be easily lost.

Information about the location of underground utilities and facilities is often worse than the maps showing above ground features. Utilities and facilities are marked on plans but are rarely up-dated. In most cases the actual location of these service lines is different from the location appearing on those plans. Agencies responsible for the provision of different infrastructure maintain their own maps usually showing only the location of infrastructure under their control. These maps are often only in the form of sketches that are interpretable only by the staff of these organizations. For underground utility lines the depth of the lines is seldom or correctly shown on maps. It is often the case for these utilities that some lines run on top of another utility line at a different depth.

These management practices are workable for general planning purposes when the size of a city is small, when land prices are low, when the land market is almost non existent, and the pace and lifestyle is slow. In these conditions, co-ordination between staff is possible and there is time to share information to overcome the anomalies in the spatial information. But for the large rapidly growing cities of developing countries today, which are becoming the engines of economic development, these management practices are woefully inadequate.

\section{Case study of the Bangkok Land Information System}

In order to assess the GIS implementation in a developing country, a case study of the Bangkok Land 
Information System (BLIS) is presented. The initial development strategy, the extent to which it partially succeeded, the reasons why it has not progressed as fast as hoped, and the lessons this provides for other developing countries, are addressed in the case study. As a result of the experiences gained in the BLIS, suggestions are made on a successful approach to implementation of a spatial information infrastructure in developing countries. An important part of the suggested approach is to provide short term, useful and low cost outputs.

\section{The BLIS project}

The City of Bangkok provides an interesting case study for examining the problems and strategies in developing an integrated spatial information system. Having realized the problem being faced by the city, the Bangkok Metropolitan Administration (BMA) decided to initiate a pilot GIS project nearly 15 years ago (Suwarnarat, 1991). BLIS was undertaken from 1989 to 1991 (Williamson and Mathieson, 1994). The primary objectives of the BLIS project were:

(1) To educate, train and provide experience to Thai Government officials in GIS;

(2) To evaluate the need for an appropriate common base map for the City of Bangkok to be used by all partners of the BLIS program; and

(3) To determine an achievable long term strategy for development of the BLIS.

These objectives were conceived on the basis that the GIS technology should integrate various agencies responsible for the management of the city. Also, the technology should be appropriate to the needs and resources available in the BLIS organizations, be relatively simple yet should be able to perform the task, be cost effective, and be application and user oriented.

Initially, a team comprising the Metropolitan Electricity Authority (MEA), the Metropolitan Waterworks Authority (MWA), the Telephone Organization of Thailand (TOT), the Department of Lands (DOL) and the BMA was put together to undertake the BLIS project with the assistance of Australian technical aid (Williamson and Mathieson, 1992, 1994).

The BLIS project initially sought to establish the requirements for a common digital map base for Bangkok. The essential data items considered necessary by each BLIS partner organization for its own purposes were identified (Suwarnarat, 1992). Although a common interest existed, there were also different points of view. The planners were satisfied with maps of scales 1:10,000 and 1:4000, whereas most of the participants from the utility agencies required a scale of 1:1000. However the TOT insisted on a detailed base map of scale 1:250. Apparently, the TOT engineers mistook the GIS as a land parcel map which they would like to use for CAD (computer aided design) to produce engineering drawings.

Such misconceptions can arise because GIS, GPS
(Global Positioning System), LIS (Land Information System), RS (Remote Sensing) and AM (Automated Mapping) are inter-related and often mistaken for each other, especially in developing countries where the technology is not as widely known or understood. This lack of understanding gave rise to protectionism and rivalry among the BLIS government agencies which were competing for budgets to develop their own computerized systems. In this environment a powerful authority with a misconception may get control over the budget and obstruct useful developments in other organizations.

There were also administrative problems in the BLIS project team due to misunderstandings of the strengths and weaknesses of GIS vis-à-vis CAD. The GIS operators were often asked to modify maps or produce visual effects which were beyond the capabilities of the system. Differences in speed, accuracy and graphic qualities combined with unrealistic expectations led to confusion among the potential end users. This situation was compounded by many of the senior personnel in the BLIS agencies having visited developed countries or international GIS conferences and seeing impressive GIS demonstrations, themselves often only pilot or demonstration projects. These expectations placed considerable pressure on the BLIS team.

After considerable discussion, various "compatibility" levels of different map scales required for urban planning and management were determined as illustrated in Table 1. Informally, a generalized description was adopted.

Having determined that 1:1000 was a suitable base mapping scale to satisfy most needs, the next step for the BLIS team was to determine the specific graphic elements and associated attributes to be included in the common BLIS database. The major factor in adopting a base map of 1:1000 was that the DOL had an excellent hard copy cadastral map for Bangkok overlaid on large scale rectified photomaps. In one sense Bangkok has one of the best urban mapping systems in the developing world as a result of the Thailand Land Titling Project ${ }^{1}$ (Angus-Leppan and Williamson, 1985a, b).

In the context of this article, a land information system is defined as a GIS which utilizes land parcels as the link to the non-graphic database attributes. The BLIS was conceived as a GIS designed primarily to serve the urban utility agencies (MEA, MWA and TOT) who share the same land information, such as the type of buildings and the name of the owners of land parcels, with the BMA. The BMA collects land

\footnotetext{
${ }^{1}$ The Bangkok Land Titling Project is a joint project by the Royal Thai Government, the World Bank and the Australian International Development Assistance Bureau. It is primarily concerned with issuing land titles to all freehold parcels in the Kingdom of Thailand, in addition to undertaking land administration reform, establishing a national valuation system, carrying out urban cadatral mapping and developing a national land information strategy.
} 
Spatial data infrastructures for cities in developing countries: I D Bishop et al.

Table 1 Base-map compatibility level

\begin{tabular}{llll}
\hline Scale & Meter/mm & Resolution & Application \\
\hline $1: 10,000$ & 10 & Some roads may disappear & $\begin{array}{l}\text { Strategic planning, regional } \\
\text { planning, city planning (GIS) } \\
\text { Tax planning (LIS), utility } \\
\text { planning and management }\end{array}$ \\
$1: 4000$ & 4 & Some houses may disappear & All the above \\
$1: 1000$ & 1 & No problems in general & CAD, engineering design \\
$1: 250$ & 0.25 & Too many map sheets & \\
\hline
\end{tabular}

tax, building tax and bill-board tax as well as a wide range of other services. While the utility agencies may not need land parcels to calculate the demand for their services or to plan for rate collection, the land parcel framework is very useful for the installation, operation and maintenance of their infrastructure. DOL was invited as a partner since it provided the base map.

The database was linked to numerous attributes with the most common information shared by the BLIS partners being the owner's name, the area, location and type of building. Other specialized items such as cable sizes, water pipe dimensions and location of meters were input and managed by participating BLIS organizations, and were not of direct concern to the BLIS project team as a whole.

It was expected that significant cost savings and an improvement in efficiency would result from sharing substantial core data sets and particularly the base map, since it was acknowledged that data acquisition would be the most important cost factor in developing GIS in the BLIS partner organizations. The reality was that the BLIS team found that the conversion of the analogue map base into digital vector maps was an expensive, difficult and time consuming operation. Attribute data were often difficult to obtain.

At the end of the project, instead of establishing a common database, the MEA (which is part of central government and has access to significant funds) took the leading role in creating a digital base map to suit their specific needs. The MEA believed that an investment in GIS would be returned almost immediately through the collection of additional tariffs. A similar route was followed in cities in many developed countries where a utility agency took a leading role, Australia for example. The MEA created its base map at a scale of 1:1000 by digitizing the DOL maps which were from the same source as those used for BLIS.

The BLIS pilot project members have now separated with each pursuing their own goals in GIS development. However they still maintain a core group of participants in various government committees concerned with GIS/LIS and AM/FM technology. A national committee for the promotion of GIS was established in Thailand in 1996. The committee resembles the strategy proposed by Williamson and Mathieson (1994) but the focus of the committee has moved to the national level. A senior BMA official chairs the subcommittee responsible for GIS/LIS data in the Bangkok region. Encouragingly, every member of the original BLIS project is represented in this subcommittee.

Surprisingly, 8 years after the initiation of the BLIS project there is still little to show in the BMA, although a great deal of experience has been gained by those involved with the project. It has been suggested by some of the former BLIS partners that the base map now produced by the MEA is not sufficiently accurate, but there is no serious challenger who is prepared to develop a more accurate map. Importantly, every member of the BLIS project has now agreed to use the MEA map. These recent developments have confirmed the benefits and strategy of the original BLIS pilot project.

After more than a decade of experimenting with GIS, the BMA now realizes that GIS is an effective tool for planning and managing urban infrastructure and utility systems. However, the vision to develop and maintain a large common digital base map within the BMA has not been realized after nearly a decade of struggle. It is now recognized as costly and administratively complicated. This has been an important lesson.

An alternative approach which some BMA officials believe has the best chance of success is the development of application oriented GIS to deal with specific issues and areas of concern. Such an approach could use a medium scale 1:20,000 base map which was available and produced by the private sector. Using this approach the benefits of the use of GIS can be seen without waiting for a large scale common base map to be established. A suitable purpose built database at a medium scale can be completed more quickly than a comprehensive or multi-purpose large scale database. The GIS application for these individual projects may be relatively simple but may provide major benefits. For example the Ministry of Industry in Thailand found a digital terrain model showing locations of BOD load sources useful to help locate waste water treatment plants (Suwarnarat et al., 1994).

The success of small strategic applications can help establish the value of GIS in developing countries. For an organization such as the BMA, the BLIS experience suggests such a strategic application oriented approach is more relevant than a system-wide 
comprehensive strategy. This may not be the ideal from a system development point of view in the medium to long term, but it is the reality. It is recognized in the context of developing countries that it is very important that the cost and investment in GIS is recovered through short term benefits as usable products in urban planning and land management. The cost benefit curve of an institutional GIS is steep i.e. the full benefits of GIS are realized often only in the long term, and in the intervening stages costs outweigh the immediate benefits to the community. There is often a misunderstanding of the respective roles of GIS infrastructure (SDI) and GIS business systems. In general, a city cannot produce GIS benefits without an investment in the underlying infrastructure. This requires a recognition that GIS infrastructure by its very nature does not return significant benefits, it is only the GIS business applications, which build on the infrastructures, which return benefits. This phenomenon is universal. To highlight the application oriented approach based on a medium scale SDI, some examples of the use of GIS in strategic management in Bangkok are presented in the following section.

\section{Strategic management in Bangkok using GIS}

The metropolitan area of Bangkok consists of 40 districts under the overall control of a Governor. In order to demonstrate the potential of spatial information handling technology in a developing country, a simple project in producing maps from existing BMA data was undertaken. Within the City of Bangkok various socio-economic and demographic data for each district are collated annually and included, as a normal practice, in a City Yearbook. Fig. 1 illustrates an example of the cartographic representation of the referred data sets.

These data had been stored as a spreadsheet and were made available along with a file (in DXF format) of the district boundaries. The boundary file had been digitized from a 1:20,000 source map.

The attribute data used include many of the normal statistics collected in most large cities on a district-bydistrict basis such as population, employment, public facilities (schools, hospitals, etc.), land use, housing pattern, factory numbers, voting patterns, land tax returns, garbage collection rates, etc.

These data have been available annually in Bangkok for the last 15 years. Until now these data have not been linked to any spatial reference or mapping system and consequently could not be fully utilized for decision making. It is the view of some of the senior BMA officials that the mapping of these data after simple analyses will not only be very useful in assisting the management of Bangkok but will also be very influential in promoting the wider use of GIS in the BMA.

Using these data, the following set of maps were produced to assist decision making on various aspects of the management of the City by the Governor of
Bangkok. Since the BMA operates according to official management plans, such as an "annual plan" and a " 5 year plan" these spatial analyses will be very useful for strategic planning. Bangkok is managed according to a 5 year plan which is prepared by officials under the supervision of the Governor and is approved by the city council for implementation. An annual plan is controlled by the annual budget and is prepared in the same way as the 5 year plan.

District officials are expected to be versatile, to know their district and their people, and to implement agreed plans efficiently and effectively. Simply a district official needs mapping and associated data at a scale of 1:1000. Department officials, on the other hand, are professional people under a "permanent secretary" or city clerk. These officials are expected to be knowledgeable and have experience and expertise in the applications area for which they are responsible. They are more concerned with long term strategic planning for which maps of $1: 10,000$ scale or smaller are sufficient.

The maps generated are thus suitable for departmental officials. Maps have been prepared relating to garbage disposal, primary education and population density, but many other similar maps based on Yearbook data could have been just as easily prepared in conjunction with digital boundaries of corresponding geographies.

Examples of maps (Figs 1-3) produced to assist in decision making are described below:

(1) Garbage disposal is one of the most costly services provided by the BMA. One person generates 500-2000 kg per year of garbage depending on their lifestyle. The disposal of garbage by incineration costs $\$ 2 / \mathrm{kg}$ but $\$ 0.2 / \mathrm{kg}$ in the case of land-fill or composting. The Governor or the appropriate senior official must know in which districts the generation of garbage and revenue are in balance. With the GIS the Governor can oversee the management of garbage disposal.

(2) According to Thai law, primary education is compulsory with the BMA being responsible for providing this service in Bangkok. Individual district chiefs of the BMA manage this service. They need to know the supply and demand situation for their district. The Governor or the appropriate senior official need to know the relative proportion of schools and school age children among all districts.

(3) The districts are of different sizes and have different populations. A GIS map with population density as an attribute is basic information indispensable for policy making by the Governor, since the annual budgets of the districts depend on it.

\section{Other BMA initiatives}

The BLIS group in the BMA is looking at new techniques to capture data for input to GIS. This includes 


\section{Specific Generation Rate of Solid Waste, 1994 Districts of Bangkok Metropolitan Area}

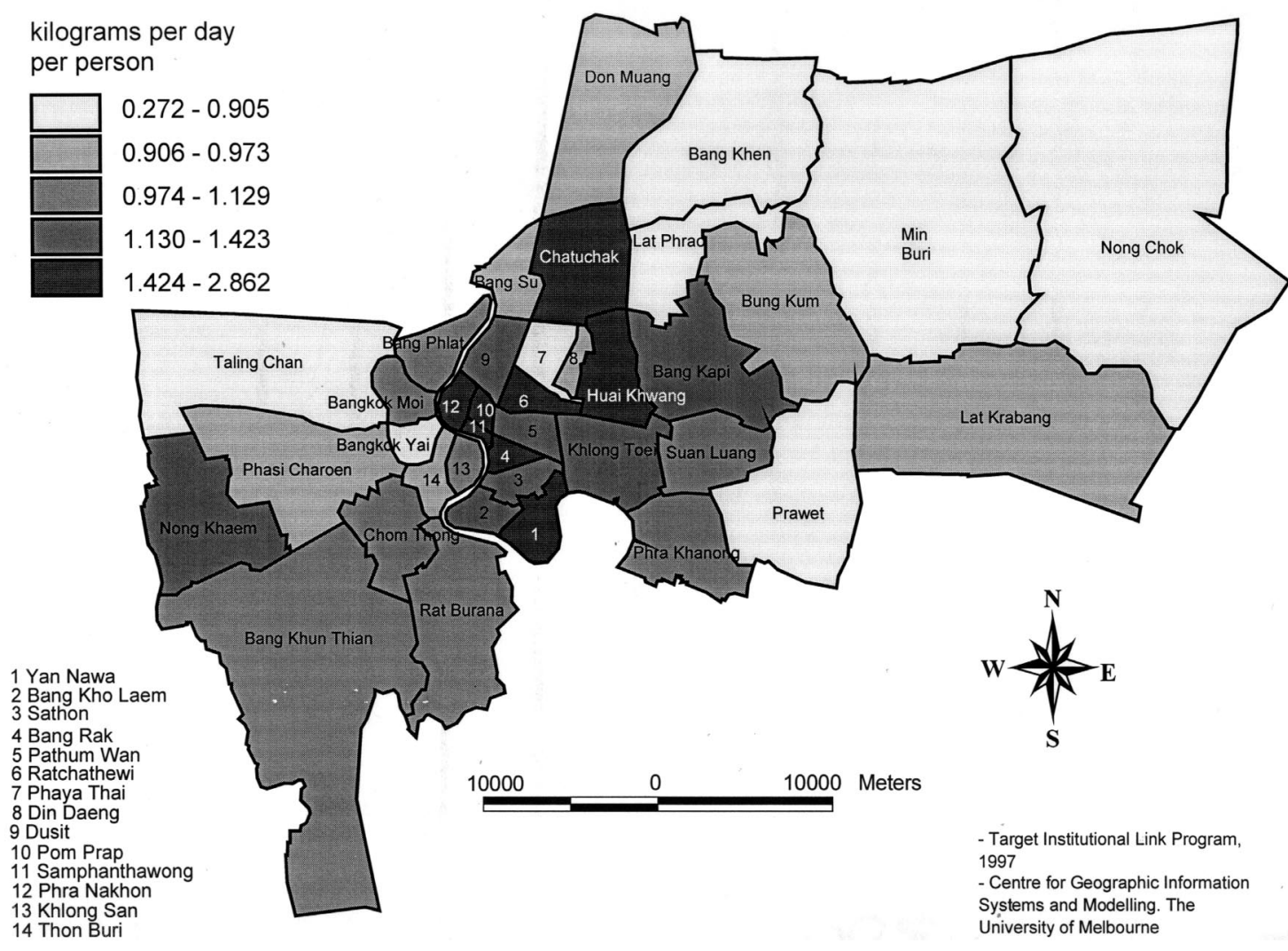

Figure 1 Generation rate of solid waste in Bangkok metropolitan area, 1994

the use of raster images and other techniques to estimate attribute data such as population (Webster, 1996). Automatic conversion of raster data to vector data has not proved to be reliable. A trial scanning of a 1:4000 map of Bangkok showed that about 30\% of the buildings did not appear as closed polygons. Much touch-up work was required. However, scanning is a quick solution for the overall view of the terrain and a broad coverage of an occurrence, such as a flood. New technologies in which resolution and ease of interpretation of remotely sensed and scanned images are emerging will broaden the applicability of these technologies (Mason et al., 1997).

Aerial photos are another type of raster data. Such data are used effectively as a background to enhance vector maps. As mentioned, the cadastral base for the DOL produced a vector land parcel map. Overlaying it on an orthoimage (rectified photomap) enables the visualization of the relationship between the parcels and the surrounding geography. In 1996, the BMA obtained a new set of aerial photographs to support planning and related activities, however they are yet to be used for security reasons. They also plan to use less expensive small format $(4 \times 4$ in.) aerial photography to produce and update maps. Again the
BMA has had this technology for many years but to date it has not been able to use it owing to internal reasons including delays in gaining approvals to take photographs for security reasons.

It is hoped that in time the BMA will utilize the large scale digital base map produced by the MEA as a result of the 10 years exposure to GIS technology, the result of the BLIS experience and as a result of the project oriented GIS described above. As a result of these experiences the BMA is in a much better position to adopt GIS technology.

\section{Spatial information development issues in developing countries}

The Bangkok experience in the development of spatial information infrastructures provides a generic framework for cities in developing countries facing a similar challenge. Although urban administration and institutional arrangements differ among countries, the issues discussed here provide an insight into the technological, managerial and institutional issues involved in the adoption of spatial information systems for cities in developing countries.

Cheaper and faster computer technology is coming 


\section{Education. BMA Pupils, 1994 Districts of Bangkok Metropolitan Area}

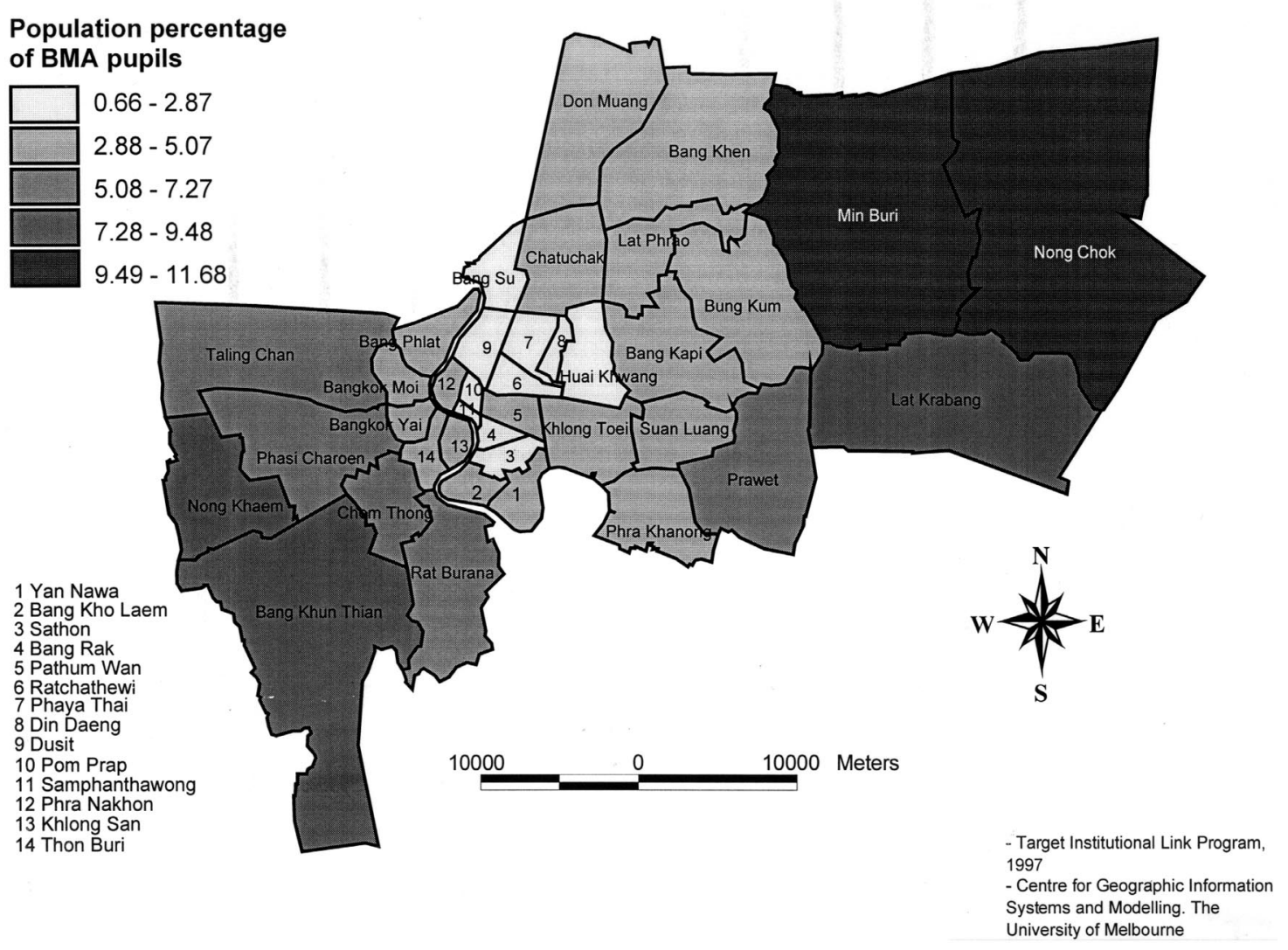

Figure 2 School age children in Bangkok districts, 1994

within the reach of most organizations in developing countries. It is therefore appropriate to consider the ways in which this technology has been implemented in developed countries - even though the implementation strategies may not be appropriate for cities in developing countries. In particular, future trends must be considered in order to understand the potential information infrastructure to which the developing world may aspire - while recognizing that different problems and conditions exist in developing countries.

There is a range of GIS implementation strategies that have been adopted in developed countries. Three main steps of planning, analysis and implementation are of importance in GIS implementation (Korte, 1994). The diffusion of GIS in an organization has been extensively discussed (Campbell and Masser, 1995) and the main phases established by the NCGIA (1990). The adoption of new spatial information technologies in developing countries should also be considered in the context of these main steps and approaches. However these approaches should be modified by taking into account the following factors which can impede the adoption of spatial information technologies in developing countries.
Need for awareness and management support

As in the developed world, the successful implementation and use of spatial information infrastructures in developing countries is dependent on political and institutional support. Management support is necessary in building the infrastructure, understanding the limitations and providing the necessary flow of information to the system. This was particularly important in maintaining the momentum of the Bangkok project over many years. The project only received the necessary political support after a Professor of Surveying was seconded from a university to the BMA as a deputy governor. On the other hand, varying awareness of GIS in an organization may bias the results. Staff will define products based on their personal awareness of GIS, on a vendor's demonstration, on a demonstration they saw at a conference or during a visit to a developed country, not on an abstract need for information. For example the BMA requested 3D perspective views of data, regardless of their actual value in decision-making processes.

\section{Data standards}

Multiplicity of agencies involved in urban management and their individual data requirements vary 


\section{Registered Population Density, 1994 Districts of Bangkok Metropolitan Area}

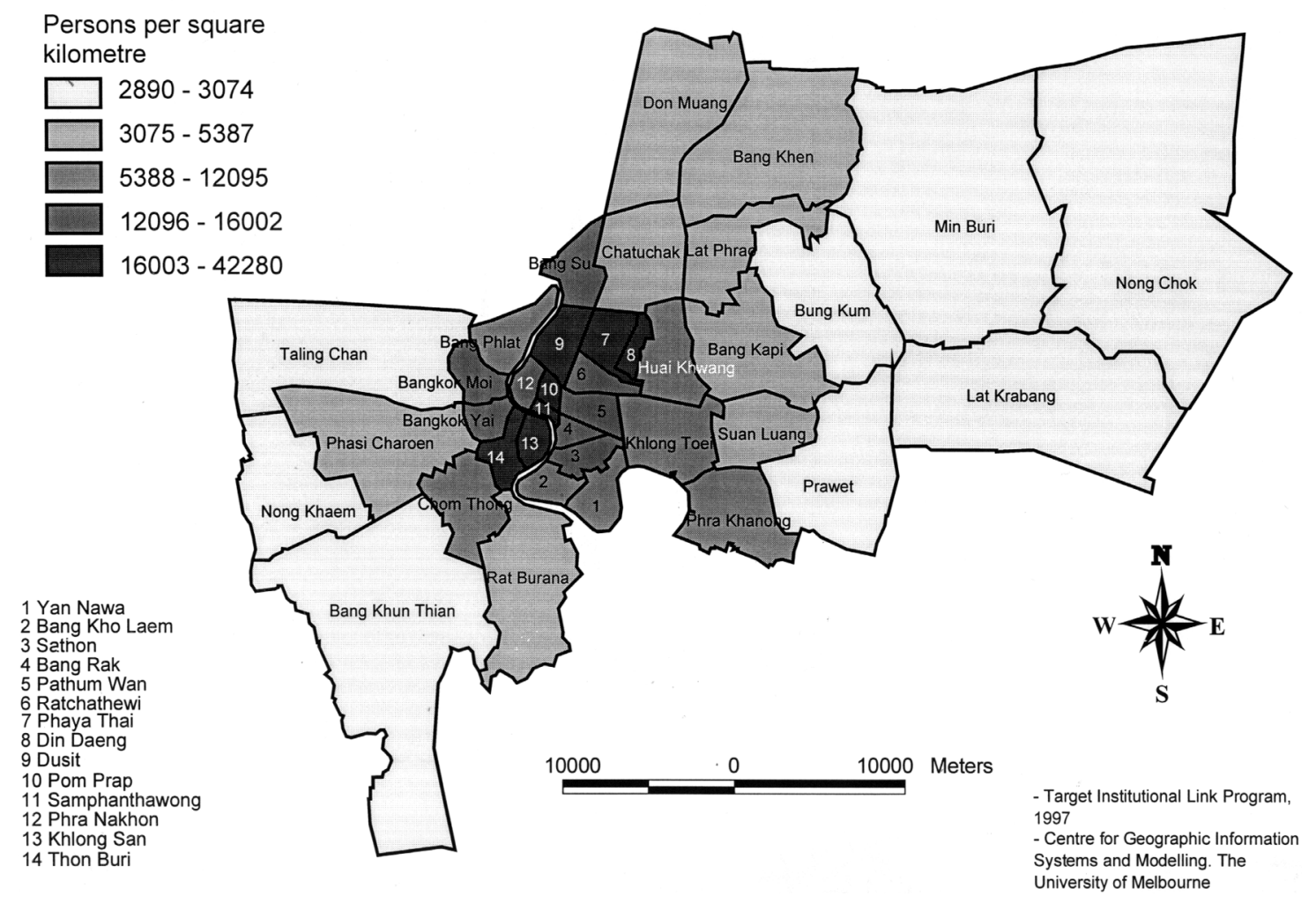

Figure 3 Registered population density in Bangkok metropolitan area, 1994

widely in terms of map scale, graphic elements, data accuracy and data currency. This makes the adoption of a common standard very difficult in any country, but especially in developing countries. A common data standard accepted by all potential users will reduce duplicity of data and data redundancies. As seen in Bangkok, utility agencies require larger scale maps than planning agencies. A map scale of 1:4000 or smaller is sufficient for strategic urban planning and urban monitoring but a scale of 1:1000 or larger is required by the utilities. An agreement about the map scale at the very beginning of spatial infrastructure development is important as it will determine the application uses. In practice it is very difficult to gain agreement due to different institutional needs.

A common practice in developed countries has been the establishment of data standards, fixed scales and data accuracy definition for national digital map bases. This task has normally been done by the National Mapping Agency (NMP). Lack of such organizations in most developing countries cause many problems in developing appropriate urban spatial information infrastructures. The BLIS is a good example of a strategy to develop appropriate standards in a developing country.

\section{Data availability}

The successful development of spatial information infrastructures in developed countries has partly been due to much of the data being available in digital form or there being a political will and financial backing to build the required datasets. However, in developing countries the situation is very different. Data are generally not available in digital form and base maps are often outdated. Even where spatial data is in digital form few results have been derived. In order to have an efficient GIS operational in developing countries it is important to start with basic data collection. This requires time which few managers either have available or are willing to commit as the benefits derived are not immediate.

There often appears to be a political urgency which negates long term planning in developing countries. For example it is often stated that the GIS must be operational in say 2 years, but since this is virtually impossible, an appropriate strategy is not implemented thereby resulting in little progress 10 years later. Conversely, developed countries built their digital systems from well established manual map systems which have often evolved over decades 
and sometimes centuries. Developing countries are becoming a good area to evaluate the potential of new high spatial resolution imagery in medium to small scale map making. By using this source of information, developing countries have the potential of saving time and money as they avoid the intermediate step of establishing manual map systems.

\section{Personnel}

There is an acute shortage of trained professional GIS personnel capable of introducing spatial information technologies in developing countries. In the case of the BMA, out of 20,000 employees there is not a single GIS professional. People unfamiliar with a new technology can also have serious concerns about being replaced by a computer and often view the technology as a competitor rather than an assistant. This was the case in developed countries in the early stages of GIS implementation processes in the public sector. Education, training programs and policies to extend the level of awareness of the technology appear to be absolutely essential and one of the best uses of international aid programs to promote GIS in developing countries. The installation of GIS hardware and software without extensive medium to long term education programs, as distinct from short term GIS training, often results in failure in developing countries.

\section{Resources}

Because of current practices in local governments in developing countries, it is often difficult to get budgets approved for the purchase of new technology, particularly when senior administration has little experience with the technology. GIS requires investment in hardware, software, data collection, data maintenance and education and training, and it is very difficult to get support for such projects from senior management without the awareness of the potential of the new technology. There are, however, low cost desk top GIS software packages, with some of them being available through the Internet at no cost. Such systems do not require specialized hardware, have simple, easily learnt interfaces, provide the essential spatial data handling procedures required of GIS software and allow rapid visualization of information in a spatial context, and particularly for parcel based data which is the key for most urban applications. In particular they are good for raising awareness and gaining experience. For many urban applications, these simple desktop low cost GIS provide an important way forward.

\section{Procedures}

The bureaucratic procedures for approval and procurement of technology in developing countries are often very cumbersome. It requires a great deal of patience and energy in many developing countries to find a way through the political system to obtain sup- port and funding, prepare tenders, evaluate tenders, award contracts and take delivery of the system. In most cases, by the time the contract is awarded, either the technology intended for use is outdated or the persons promoting such projects are transferred (and often both!). As a consequence, the project is often delayed. All through the developing world there are GIS and related technologies either under-utilized or lying idle for these reasons.

\section{Vendor support}

GIS is a new field with a limited clientele in many developing countries. Often there is little vendor support for GIS software. Often, monopolistic conditions exist for a vendor who provides software and after sales support. This monopolistic phenomenon often results in higher costs, poor services and limited expertise. It follows that many government departments do not want to invest in sophisticated GIS products. It is often cheaper and easier to implement simple off-the-shelf packaged software in developing countries.

\section{Successful implementation}

Unfortunately there are few successfully implemented GIS projects in developing countries that provide guidance to others. The establishment of spatial data infrastructures and GIS is very difficult without a great deal of commitment from all involved. However, there are many cases where spatial data handling technology has been introduced in developing countries by developed countries in the form of international aid. It is important for the successful implementation of GIS in a country (developed or not) that the technology used should be well known and the people using it be well educated and trained in its use. Successful systems will need to manage with little initial data and lots of initial skepticism from the organization. Systems should have a smooth learning curve, be relatively simple to learn and operate (measured in days not months), an ability to perform important basic functions with ease and easy database building capabilities. Such systems should be capable of integrating and accessing existing data sources.

\section{Data integration}

It is increasingly recognized that linkages between the various spatial information development areas leads to greater applications and benefits. Increasingly access to all these capabilities will be via a single interface, across an Intranet or the Internet, giving access to different features provided by a variety of software products. In future, data from all platforms will be conveniently exchanged and interpolated for testing decision scenarios. Integrated systems will provide seamless decision support based on shared data using various forms of predictive modeling. Results will be presented through a multimedia inter- 
face and include direct comparison of results in a range of accessible formats, from spread sheets to maps, to photo-realistic visualization of relevant outcomes. The presentation options will be suitable for the wide range of disciplines involved in urban planning and design.

These issues are the focus of current research in developed countries and are at an early stage of implementation. However, developing countries have the opportunity to learn from developed countries' mistakes to start their own process avoiding those mistakes. The rapid advances in network based data access (Buttenfield and Kumler, 1996), modeling and visualization (Pesce, 1995) suggest that the necessary integration tools are already here.

In coming years, a vast majority of the workforce in developed countries will become proficient in the use of these new technologies which will have a major application in urban administration and management. Powerful computers with imbedded multimedia and GIS capabilities will be available as a common household tool. However this scenario will not necessarily occur in the developing world. The International Conference of the Internet Society, INET'98 (Geneva) has highlighted this issue as one of the main concerns in developing countries regarding technology diffusion (Hargittai, 1998). This has important implications for their adoption strategies.

The above technologies obviously have great potential in supporting improved urban management and administration. However the experience of developing countries, even with the most basic spatial information technologies, has not been good, for the reasons explained above. As such, care should be taken by developing countries in adopting the latest technologies which are only now being adopted in the developed world.

\section{Conclusion}

Cities in the developing world are expanding at a much greater rate than in developed countries with resulting major environmental and management problems. While these cities are usually the engines of economic development in the respective countries, their infrastructure and quality of life is often deteriorating. Addressing these issues in cities of the developing world is one of the great challenges facing all societies in the next millennium.

GIS and the underlying spatial data infrastructures and technologies being adopted in the cities of the developed world have the potential to help resolve many of these urgent issues and problems in the developing world. However care is required. The economic, social, institutional, legal and technical environment in the developing world is very different from that in the developed countries. As such the use of GIS and related technologies has had a chequered history in developing countries with many systems being under utilized or lying idle.
By reviewing the environment in developing countries, this paper has attempted to understand and explain the problems and issues in applying spatial information technologies, often taken for granted in the cities of the developed world. The paper reviews the Bangkok Land Information System project as an example to highlight the issues and to suggest some generic strategies.

The main conclusion from this research is that the development of a digital large scale parcel based map as basic spatial infrastructure for a range of GIS business applications is very difficult to achieve for many countries in the short to medium term. The main limitations are a lack of appreciation of what GIS can and cannot do, lack of resources and trained personnel, inefficient bureaucratic processes, lack of data, and lack of hardware and software vendor support.

In addition, it is suggested that small to medium scale projects or business based GIS are the best option to introduce the GIS concept to cities, to gain acceptance of the technology, as is shown by the Bangkok examples in the paper. In this regard, high resolution satellite imagery offers an opportunity to obtain the basic spatial data infrastructure or digital map base required to support small to medium scale urban GIS in the future.

It is acknowledged that the development of technology is moving at a rapid pace, and that while the above suggestions are considered appropriate in the foreseeable future, based on current technology, it is quite likely that new or improved spatial technologies will arise. This could have direct application in the cities of the developing world, even though personnel, institutional and financial issues will continue to constrain adoption.

\section{Acknowledgements}

This paper is the result of a continuing association between the University of Melbourne and the Bangkok Metropolitan Administration. That association has received support under the Targeted Institutional Links Program of the Department of Education, Employment, Training and Youth Affairs (DEETYA) of the Federal Government of Australia. In addition Khun Samphet of Chulalongkorn University is acknowledged for providing valuable assistance in the conversion of data and the preparation of maps.

\section{References}

Angus-Leppan, P V and Williamson, I P (1985a) A project for upgrading the cadastral system in Thailand Part 1. The Survey Review 28(215), 2-14.

Angus-Leppan, P V and Williamson, I P (1985b) A project for upgrading the cadastral system in Thailand Part 2. The Survey Review 28(216), 63-73.

Buttenfield, B P and Kumler, M P (1996) Tools for browsing environmental data: the Alexandria digital library interface. Third International Conference on GIS and Environmental Modeling, Santa Fe, CA. 
Spatial data infrastructures for cities in developing countries: I D Bishop et al.

Campbell, H and Masser, I (1995) GIS and Organizations. Taylor and Francis, London.

Hargittai, E (1998) Globalization and regional implications: the Internet and global socio-economic development. INET'98, Geneva, 21-24 July.

Korte, G B (1994) The GIS Book. Onword Press, Santa Fe, CA.

Lai, P-C and Wong, M (1996) Problems and prospects of GIS development in Asia. GIS in Asia, ed. Tung Fung, Poh Chin Lai, Hui Lin, Anthony Gar-on Yeh. GIS Asia Pacific, pp. 195199. Hong Kong.

Mason, S O, Baltsavias, E P and Bishop, I D (1997) Spatial decision support systems for the management of informal settlements. Computers, Environment and Urban Systems 21(3-4), 189-209.

NCGIA (1990) Core Curriculum. Vol. I: Introduction to GIS. Vol. II: Technical Issues in GIS. Vol. III: Application Issues in GIS. National Center for Geographic Information and Analysis, University of California, Santa Barbara, CA.

Pesce, M (1995) VRML Browsing and Building Cyberspace. New Riders Publishing, Indianapolis, Ind.

Suwarnarat, K (1991) BLIS, a new tool for the urban development. First International Conference and Exhibition in Thailand on CAD-CAM-CAE-CIM-AEC-GIS Technologies and Application, Bangkok, July.
Suwarnarat, K (1992) An improved information system for the urban development of Bangkok. ENTECH 1992 SEMINAR, Sirikit Convention Hall, Bangkok, 18 October.

Suwarnarat, K, Limpongsanuraks, S, Pumpuang, A, Chartmethakul, S and Chartmethakul M (1994) The application of GIS in the control of water pollution. CATT 94 SEMINAR, Sirikit Convention Hall, Bangkok, 27-29 June.

United Nations (1995) World Urbanization Prospects: The 1994 Revision. United Nations, New York.

Webster, J C (1996) The potential of urban texture measures in monitoring urbanization from space. GIS in Asia, Asia GIS/LIS,AM/FM and Spatial Analysis Conference, Hong Kong, pp. 309-322. GIS Asia Pacific Hong Kong Geographic Association, Hong Kong.

Williamson, I P (1991) Land information management at the World Bank. The Australian Surveyor 31(1), 41-51.

Williamson, I P and Mathieson, G (1992) The Bangkok Land Information System Project - designing and integrated land information system for a large city in the developing world. CISM Journal 46(2), 153-164.

Williamson, I P and Mathieson, G (1994) The Bangkok Land Information System - past and future. The Australian Surveyor 38(4), 298-307. 